\title{
Development of a Comprehensive Sequencing Assay for Inherited Cardiac Condition Genes
}

\author{
Birgit H. Funke ${ }^{1,2}$
}

Received: 20 December 2015 / Accepted: 21 December 2015 /Published online: 6 January 2016

(C) Springer Science+Business Media New York 2016

Clinical genetic testing for inherited cardiac conditions (ICC) began in the late twentieth century and has since rapidly expanded from single gene testing via Sanger sequencing to routinely interrogating large numbers of genes via nextgeneration sequencing (NGS). Catalyzed by the disruptive innovation of NGS, major paradigm changes have taken place in the medical genetics field. Historically, genetic tests were configured for a single disorder and testing was typically performed after a clinical diagnosis had been established. As genetic testing is more widely adopted in the clinic, it has been increasingly recognized that the phenotypic spectrum of many disorders is larger than appreciated and that sequencing a broader array of genes covering related clinical entities can aid in establishing or refining a clinical diagnosis [1]. For this reason, large multi-disease gene panels are now frequently utilized for disorders with clinical and genetic heterogeneity including many inherited cardiac conditions. For example, gene panels for dilated cardiomyopathy (DCM) now typically also include genes associated with hypertrophic cardiomyopathy (HCM) and arrhythmogenic right ventricular cardiomyopathy (ARVC) as these disorders can be the primary genetic etiology in a patient with DCM [2]. Similarly, sequencing tests for Noonan syndrome initially included only Noonan syndrome genes but today cover overlapping rasopathies [3].

Editor-in-Chief Jennifer L. Hall oversaw the review of this article

Birgit H. Funke

bfunke@partners.org

1 Department of Pathology, Massachusetts General Hospital and Harvard Medical School, Boston, MA, USA

2 Laboratory for Molecular Medicine, Partners HealthCare|Personalized Medicine, 65 Landsdowne Street, Cambridge, MA 02139, USA
With NGS prices continuing to drop, molecular genetic testing laboratories are beginning to adopt whole-exome and whole-genome sequencing (WES, WGS). Using those assays as a first-line genetic test seems to be within reach, which has recently spurred a debate as to what NGS test is appropriate for which clinical scenario. WES and WGS have thus far been mostly used for patients with complex diagnoses or for patients where other testing options yielded no result. However, these assays still have important drawbacks such as incomplete coverage of critical genes. This tends to be a lesser problem for most gene panels, which may therefore still be the better choice for patients with a well-established diagnosis where complete coverage of the main disease associated genes is paramount.

In this issue of the journal, Pua et al. describe a targeted gene capture panel covering 174 genes for the most prevalent inherited cardiac conditions (including arrhythmias, cardiomyopathies, aortopathies, and hyperlipidemias). Genes were selected to cast a wide net and include well-known disease genes and also genes whose disease association is less well established at this point. An in-depth curation of the level of evidence behind each gene is beyond the scope of this work but is being addressed by expert-driven community efforts such as the Clinical Genome Resource (ClinGen) [4]. The authors describe the analytic performance of this panel using Illumina's Nextera capture assay followed by sequencing on the widely used MiSeq $(\sim 300 \times$ mean depth $)$ in addition to the NextSeq500 instrument $(\sim 600 \times)$. Additionally, the completeness of coverage of the main disease genes was compared to standard WES $(\sim 70 \times$ mean depth), deep WES $(\sim 500 \times)$, and WGS $(\sim 70 \times)$. At a very high analytical accuracy, their panel clearly (and expectedly) outperformed WES and WGS in terms of completeness of the main disease genes though this difference was less pronounced for deep WES and WGS.

Part of the debate on whether exome sequencing can soon replace gene panels has focused on the increasing rate of disease gene discovery our community has been experiencing, 
making static gene panels potentially vulnerable to being outdated quickly. On the other hand, it is important to point out that few newly discovered disease genes are immediately supported by a level of evidence that warrants inclusion in clinical diagnostic tests. For most genes, it takes years until the initial discovery is replicated and supported by additional lines of evidence and many represent a rare cause of disease. WES can be a viable alternative to targeted panels, particularly if completed by Sanger fill-in of important disease genes to ensure complete coverage. However, for most diagnostic laboratories, the implementation of WES or WGS assays, let alone their complementation with Sanger fill-in approach assays, is currently still out of reach. Well-designed comprehensive gene panels such as the one developed by Pua et al. are therefore a viable and adequate testing approach for genetically and clinically heterogeneous disorders for the near-term future.

\section{References}

1. Yu, H., \& Zhang, V. W. (2015). Precision medicine for continuing phenotype expansion of human genetic diseases. BioMed Research International, 2015, 745043. doi:10.1155/2015/745043.

2. Pugh, T. J., Kelly, M. A., Gowrisankar, S., Hynes, E., Seidman, M. A., Baxter, S. M., Bowser, M., Harrison, B., Aaron, D., Mahanta, L. M., Lakdawala, N. K., McDermott, G., White, E. T., Rehm, H. L., Lebo, M., \& Funke, B. H. (2014). The landscape of genetic variation in dilated cardiomyopathy as surveyed by clinical DNA sequencing. Genetics in Medicine, 16(8), 601-608.

3. Lepri, F. R., Scavelli, R., Digilio, M. C., Gnazzo, M., Grotta, S., Dentici, M. L., Pisaneschi, E., Sirleto, P., Capolino, R., Baban, A., Russo, S., Franchin, T., Angioni, A., \& Dallapiccola, B. (2014). Diagnosis of Noonan syndrome and related disorders using target next generation sequencing. BMC Medical Genetics. PMID: 24451042

4. https://www.clinicalgenome.org/about/working-groups/clinicaldomain/cardiovascular/ (accessed 12.19.15). 